\title{
Infection and Re-Infection: Stability of Complex Air Transport Network
}

\author{
Mengbang Zou \\ School of Aerospace, Transport and \\ Manufacturing \\ Cranfield University \\ Bedford, UK \\ m.zou@cranfield.ac.uk
}

\author{
Weisi Guo \\ School of Aerospace, Transport and \\ Manufacturing \\ Cranfield University \\ Bedford, UK \\ weisi.guo@cranfield.ac.uk
}

\begin{abstract}
Global epidemic propagation rate and structure are strongly coupled with the international air transport network. Due to the network structure, countries are desynchronized in the way infections arise, transported, mitigated, and re-infect again. A global lockdown is detrimental to the international economy, and many argue that unless the whole world is evenly vaccinated, we cannot return to pre-COVID lives. The current challenge is that new waves of re-infection are spreading, and vaccination will take many months to materialise across the world. Here, we show how certain small airports $(0.1 \%$ of global) contribute much more to the epidemic spread process, irrespective of the actual spreading mechanics. We use trophic coherence as a metric for network stability in directed graphs (e.g., recurring network source of reinfection). We find that the air transport network has a trophic coherence similar to a random expectation (99\% of airports form a tightly looped network) and practically all networks need to be equally protected to ensure global security.
\end{abstract}

Index Terms-air transport network; network science; data analysis; epidemics; COVID-19;

\section{INTRODUCTION}

Future globally connected smart cities need to be conscious of the benefits and risks of domestic and international air travel [1]. In this paper, we focus on network science analysis of transport networks [2], [3], which can yield insights into the risk of not only pandemic spreading, but also waves of reinfections. In particular, we focus on the general stability of a global network to some dynamic process.

Epidemic spreading along air transport networks is a wellestablished research area [4]. Evidence particular points to the local air transport network at a virus outbreak location [5] as key to its initial spread, as well as long-range air travel on the general global epidemic development. The effectiveness of air travel to spread infectious diseases is also exasperated by the congested nature of airports [6]. Existing works approach epidemic modeling from 2 perspectives.

\section{A. Epidemic and Dynamic Process Network Models}

In the first category, evidence driven research [5] focus on how new strains or new outbreaks propagate along local air

This paper is partly funded by: EPSRC Centre for Doctoral Training in Urban Science and Progress (EP/L016400/1) and Chinese Scholarship Council (CSC). travel links from the source of the outbreak, which sets the important initial conditions of an outbreak. Any SIS/SIR/SEIR modeling [7] depends on the initial conditions and measurement accuracy to predict the success of the epidemic process [8], [9].

In the second category, more theoretical models that use network science models to predict the spread-ability of an epidemic, premised on the graph attributes of the network. Network science has been used to drive understanding in a wide range of phenomenons, using network statistics [2], community analysis [10], or node level centrality analysis [11]. In epidemics, early analysis examined Markovian dynamics driven by the largest eigenvalue of the connectivity matrix [12], but is based on Markovian dynamic assumptions and a random null network model [13]. Other work look at relatively simple local graph measures such as node degree [11] and focus on single connection impact. Expanding to a more diverse set of node centrality measures such as betweenness and assortativity was still implying dynamics that are based on shortest-paths or random walkers, and does not address the dynamics in either a direct manner or in an agnostic manner [14]. Neither do these analysis consider the stability of a new infection, e.g., how fast will it die out on the air transport network.

\section{B. Trophic Coherence: Dynamic Agnostic Network Models}

Trophic coherence is inspired by ecology as a way to reorganise the network and understand how processes spread on the network. In ecology, it is frequently used for food webs [15], [16], and has been extended to consider neuron signals to epidemics [17], as well as rail transport [18] and water distribution [19]. This framework was generalised to the spread of dynamical processes and infections [20]. The underlying ideas is that low trophic level nodes (basal) have a strong effect on the spread process, as well as the overall coherence of the network. Coherent networks are stable and can synchronize faster, whereas incoherent networks are unstable and infections and re-infections can occur in waves. 


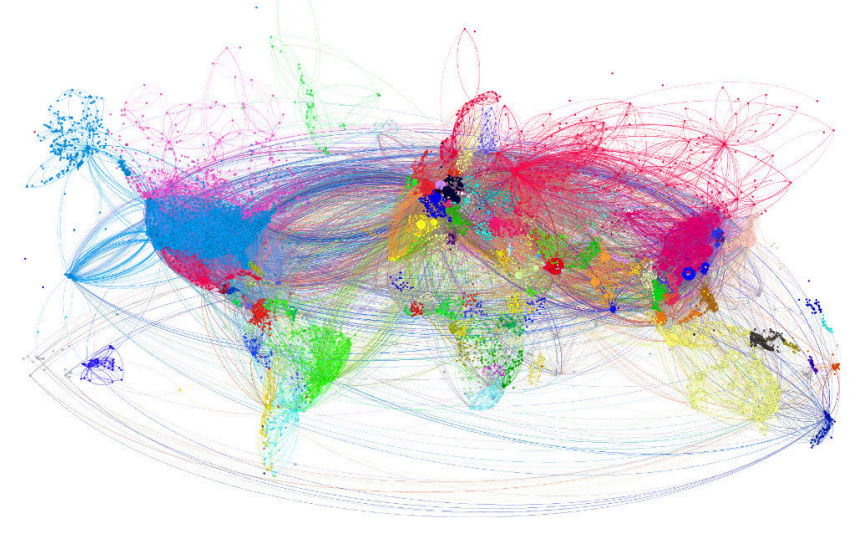

Fig. 1. Global Air Transport Network Pre-COVID19. Nodes with the same color represents airports in the same country. It is a densely looped network, while some airports disconnect with others.

\section{Innovation and Assumptions}

In this paper, we analyse the pre-COVID air transport network as a proxy to what state of travel the world wants to return to as it recovers. Yet, because there will be new COVID strains and remnants, we examine which airports are most prone to reinfecting the world. Instead of being data-driven by unknown future breakouts, we take the perspective that any breakout at global scale must be driven by basal nodes, e.g., those airport nodes that transmit the most energy from a network science perspective. This differs it from existing centrality analysis that does not consider feedback loops and network coherence and stability.

To achieve this, we for the first time, convert the preCOVID19 air transport network into a trophic network. This way we can see if the world recovers to the original state, what the challenges will be. We have the following key contributions and their associated assumptions:

1) Dynamic Agnostic Stability: the overall stability of the network for any dynamic process (e.g., we do not assume any SIS/SIR model, but rather look for general network patterns)

2) Key Airports that Drive Reinfection: identify which airports are basal nodes that drive the reinfection process, assuming that the process is already spreading (e.g., we are not looking for initial conditions)

Our work is dynamic agnostic and will demonstrate how waves of infections can occur. This sets it aside from existing work that focuses on snapshots of evidence [5], simple network centrality measures [11], [14], or dynamic specific models that assume a particular Markovian, SIS/SIR, or random walker model [13].

\section{DATA \& METHOD}

\section{A. Data Set}

We take a snapshot of the air transport network in 201718 as a representative network showing major inter-city connections. The air transport data was purchased from the commercial vendor OAG, an air travel intelligence company, and network level data (flights per airport) was calculated. Domestic flights account for approximately $50 \%$ of these flight paths. The network which we constructed using this OAG data is shown in Figure 1. We have used this data before in previous papers [2], [21]. Whilst we could have used a yearby-year analysis, we felt this was over analysing the problem as the basic network does not change significantly year by year. The data consists of flight volumes between airports, with $9000+$ airports and 34000+ flight links between them for the year that we focus on.

\section{B. Trophic Structure of Air Transport Network}

The trophic level of an airport node $s_{i}$ is defined in terms of the average trophic level of its in-neighbours

$$
s_{i}=1+\frac{1}{k_{i}^{\mathrm{in}}} \sum_{j} a_{i j} s_{j} .
$$

where $a_{i j}$ is the adjacency matrix of the air transport network and $k_{i}^{\text {in }}=\sum_{j} a_{i j}$ is the in degree. Basal nodes $k_{i}^{\text {in }}=0$ have trophic level $s_{i}=1$ by convention. By solving the system of equations (1), it is always possible to assign a unique trophic level to each node as long as there is a least one basal node, and every node is on a directed path which includes a basal node [15]. In our study the trophic level of an airport is the average level of all the airports from which it receives passengers plus one. For this reason, airports in or near remote areas tend to have a lower trophic level than those close to heavily populated coastal areas.

Each edge has an associated trophic difference: $x_{i j}=$ $s_{i}-s_{j}$. The distribution of trophic differences, $p(x)$, always has mean 1 , and a network will be more trophically coherent the smaller the variance of this distribution. We can measure trophic coherence with the incoherence parameter $q$, which is simply the standard deviation of $p(x)$ :

$$
q=\sqrt{\frac{1}{L} \sum_{i j} a_{i j} x_{i j}^{2}-1}
$$

where: $L=\sum_{i j} a_{i j}$ is the number of connections (edges) between the stations (nodes) in the network. A perfectly coherent network will have $q=0$, while a $q$ greater than 0 indicates less coherent networks. The degree to which empirical networks are trophically coherent can be investigated by comparing with a null mode. The basal ensemble expectation $\widehat{q}$ is a good estimation for finite random networks [16], which can be calculated by

$$
\widehat{q}=\sqrt{\frac{L}{L_{B}}-1},
$$




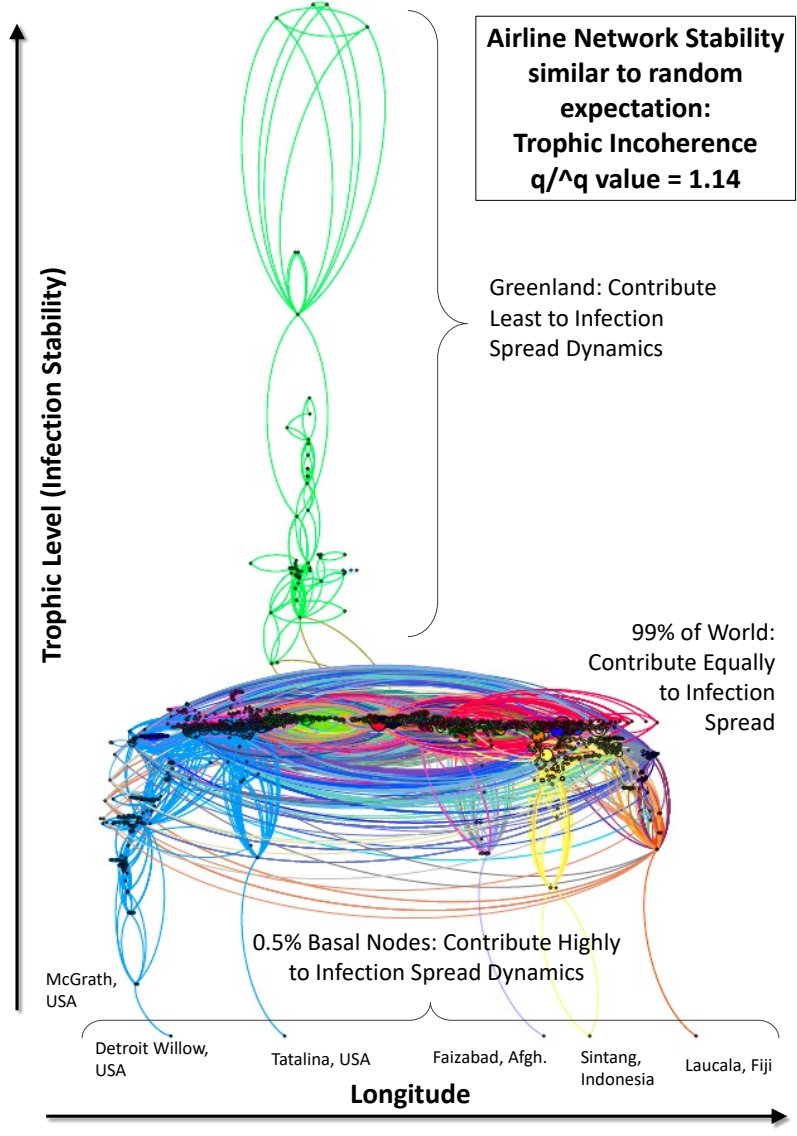

Fig. 2. Global Air Transport Network Restructured into Trophic Levels (Stability Contribution): 5 basal nodes contribute highly to network instability, 99\% of network contribute similarly, and Greenland have highest trophic level (contribute to instability the least).

where $L_{B}$ is the number of edges connected to basal nodes. $q / \widehat{q}$ can be used to analyze the coherence of the network. $q / \widehat{q}>1$ indicates that the network is incoherent (such as metabolic networks); $q / \widehat{q}<1$ reveals coherent network (such as food webs); $q / \widehat{q}$ close to 1 shows that the coherence of the network is similar to a random expectation (such as gene regulatory networks) [16].

\section{RESULTS}

\section{A. Stability from Trophic Coherence}

The major flows of passengers (from one airport to another) determine the stability of the air transport network. We can consider the flows filter method to remove the irrelevant edges. For example, the number of passengers from airport $i$ to $j$ is much higher than that from $j$ to $i$, then the edge $a_{j i}$ can be ignored. We defined the threshold $T$ to remove the irrelevant edges. If $a_{i j} / a_{j i}>T$, then $a_{j i}$ is removed. Since, the ratio $a_{i j} / a_{j i}$ of most edges in air transport network is close to 1 , the larger the $T$, the less edges will be removed. If $T$ is close to 1 , a large number of edges will be removed in the air transport network and some important information will be lost. The trophic levels of the air transport network are shown in Figure

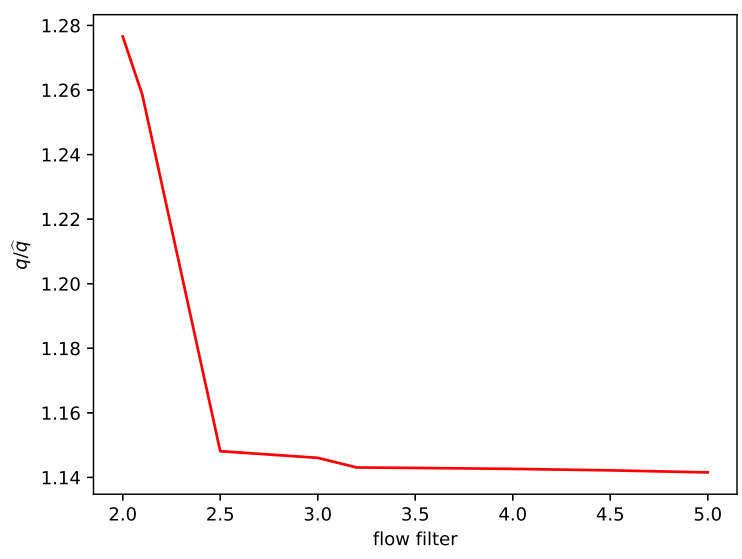

Fig. 3. The trophic coherence of the air transport network with different threshold $T$.

(2). There are 5 basal airports in the network, which mainly send passengers to the world. On the other hand, airports in Greenland have high trophic levels, which means they have little effects on the dynamics.

To analyse the stability of the network, we calculate the trophic coherence of the network with different threshold $T$. In Figure (3), it shows that when $3<T$, the ratio $q / \widehat{q}$ is robust, which provides a good condition to analyze the network. $q / \widehat{q}$ of the air transport network equals to 1.14. While, when $T$ is close to 1 , the ratio $q / \widehat{q}$ varies, even though not too much. This is because a small threshold $T$ removes a large number of edges, which cause the structure change of the network. The analysis reflects the fact that remove some loops of the network will not heavily affect the trophic coherence, which means these edges have little effects on the stability of the network.

\section{B. Airports with Low Trophic Level}

Here we set $T=3$ and the trophic levels are shown in Figure (2). There exists 5 important airports (see Figure 2) in the air transport network, which are regarded as the basal nodes. To analyse the effects of the basal nodes, we remove some of them and calculate the trophic coherence (shown in Figure 4). It shows that the removal of basal nodes will largely affect the trophic coherence of the network, which makes the network less coherent. Comparing with the removal of edges (shown in Figure 3), the removal of basal nodes have much more influence on the trophic coherence as well as the dynamics. Also, we compare the effect of removal basal nodes and nodes in other trophic levels on trophic coherence in Figure 4. The removal of basal nodes has obvious effects on trophic coherence, while the removal of nodes in other trophic level has little effects on trophic coherence. That is to say, these 5 airports are considered basal and largely contribute to infection dynamics. The research [17] shows that trophic coherence affects the infection of the network. In coherent network, the infection spreads easily in the whole network, 


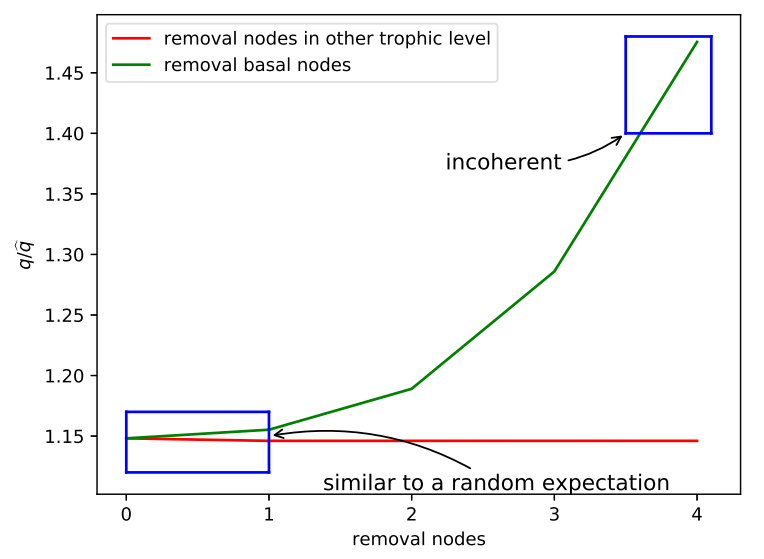

Fig. 4. The effects of removal nodes on the network. The green line shows that the removal of basal nodes cause trophic incoherence. The red line shows that the removal of nodes in other trophic level has little effects on trophic coherence.

while infection only reaches a fraction of the network in a less coherent network. Therefore, comparing with airports in other trophic levels, protecting these 5 airports is more efficient to prevent the spreading of intections in the whole network.

\section{Conclusions}

Future globally connected smart cities need to balance the benefits and risks of air travel [1] [22]. In this paper we analyse the pre-COVID air transport network as a proxy to what state of travel the world wants to return to as it recovers. Yet, because there will be new COVID strains and remnants infection pockets, we examine which airports are most prone to re-infecting the world, given no emerging variant geographic bias. Instead of being data-driven by unknown future variant breakouts, we take the perspective that any sustained re-infection process must be driven by basal nodes, e.g., those small proportion $(0.1 \%)$ of airport nodes that transmit outwards the most passengers. What we found that the trophic coherence of the air transport network is close to 1 - similar to a random expectation. Airports in Greenland have high trophic level, which have the smallest effects on the infection dynamic stability. Also, in line with intuition, due to the largely symmetric nature of passenger flows, $99 \%$ of airports have similar contribution to stability, whereas only 5 minor airports are considered basal and contribute as potential sources to unstable reinfection dynamics. As such, outside this minority, there is no clear priority order to safeguard airports and $99 \%$ of airports are equally important. The analysis of trophic coherence of the air transport network points out the important airports which contribute much more to the infection dynamic stability. Also, the whole network is divided into different trophic levels where nodes in lower trophic level affect more on the dynamic stability. The limitation is that the contribution of group or community structure in the same trophic level to the global dynamic stability is still not clear.
In the future, it is interesting to reveal the effects of group or community structure on the dynamic stability of the work.

\section{REFERENCES}

[1] R. A. Briseño, J. C. López, R. M. Arellano, V. M. Larios, J. B. Ramirez, and C. López-Zaragoza, "Digital platform to promote sustainable mobility and covid-19 infections reduction: a use case in the guadalajara metropolitan area," in IEEE International Smart Cities Conference (ISC2), 2020, pp. 1-8.

[2] W. Guo and B. T. et al., "Global air transport complex network: multiscale analysis," SN Applied Science, vol. 680, 2019.

[3] W. Guo and X. Lu, "London underground: Neighbourhood centrality and relation to urban geography," in IEEE International Smart Cities Conference (ISC2), 2016, pp. 1-7.

[4] J. Li, T. Xiang, and L. He, "Modeling epidemic spread in transportation networks: A review," J. Traffic Transp. Eng., vol. 8, 2021.

[5] G. Lawyer, "Measuring the potential of individual airports for pandemic spread over the world airline network," BMC Infectious Diseases, vol. 16, 2015.

[6] A. Ceria, K. Kostler, R. Gobardhan, and H. Wang, "Modeling airport congestion contagion by heterogeneous SIS epidemic spreading on airline networks," PLOS One, 2021

[7] M. Small and D. Cavanagh, "Modelling strong control measures for epidemic propagation with networks - a covid-19 case study," IEEE Access, vol. 8, pp. 109719-109731, 2020.

[8] R. Vizuete, P. Frasca, and F. Garin, "Graphon-based sensitivity analysis of sis epidemics," IEEE Control Syst. Lett., vol. 4, no. 3, pp. 542-547, 2020.

[9] G. Dimitriu and V. L. Boiculese, "Sensitivity study for a seit epidemic model," in 2015 E-Health and Bioengineering Conference (EHB), 2015, pp. 1-4.

[10] L. Orr, S. Chapman, J. W. Gjerloev, and W. Guo, "Network community structure of substorms using SuperMAG magnetometers," Nat. Commun., vol. 12, 2021.

[11] X. Sun, S. Wandelt, and A. Zhang, "On the degree of synchronization between air transport connectivity and COVID-19 cases at worldwide level," Transp. Policy, vol. 105, 2021.

[12] M. Boguna and R. Pastor-Satorras, "Epidemic spreading in correlated complex networks," Phys. Rev. E, vol. 66, 2003.

[13] R. Pastor-Satorras and A. Vespignani, "Epidemic dynamics and endemic states in complex networks," Phys. Rev. E, vol. 63, 2001.

[14] X. Sun, S. Wandelt, and A. Zhang, "How did COVID-19 impact air transportation? A first peek through the lens of complex networks," $J$. Air Transp. Manag., vol. 89, 2021.

[15] S. Johnson, V. Dominguez-Garcia, L. Donetti, and M. Munoz, "Trophic coherence determines food-web stability," Proc. Natl. Acad. Sci. U.S.A., vol. 111, pp. 17923 - 17928, 2014.

[16] S. Johnson and N. Jones, "Looplessness in networks is linked to trophic coherence," Proc. Natl. Acad. Sci. U.S.A., vol. 114, pp. 5618 - 5623, 2017.

[17] J. Klaise and S. Johnson, "From neurons to epidemics: How trophic coherence affects spreading processes," Chaos, vol. 26, 032016.

[18] A. Pagani, G. Mosquera, A. Alturki, S. Johnson, S. Jarvis, A. Wilson, W. Guo, and L. Varga, "Resilience or Robustness: Identifying Topological Vulnerabilities in Rail Networks," R. Soc. Open Sci., vol. 6: 181301, 2019.

[19] A. Pagani, F. Meng, G. Fu, M. Musolesi, and W. Guo, "Quantifying Resilience via Multiscale Feedback Loops in Water Distribution Networks," J. Water Resour. Plan. Manag., vol. 146, 2020.

[20] G. Moutsinas, C. Shuaib, W. Guo, and S. Jarvis, "Graph hierarchy: a novel framework to analyse hierarchical structures in complex networks," Sci. Rep., vol. 11, 2021.

[21] W. Guo, M. D. Vecchio, and G. Pogrebna, "Global network centrality of university rankings," R. Soc. Open Sci., vol. 4, 2017.

[22] D. G. Costa and J. P. J. Peixoto, "Covid-19 pandemic: a review of smart cities initiatives to face new outbreaks," IET Smart Cities, vol. 2, no. 2, pp. 64-73, 2020. 
$2021-10-15$

\section{Infection and re-infection: stability of complex air transport network}

Zou, Mengbang

IEEE

Zou M, Guo W. (2021) Infection and re-infection: stability of complex air transport network. In: 2021 IEEE international Smart Cities Conference (ISC2), 7-10 September 2021, Virtual Event https://doi.org/10.1109/ISC253183.2021.9562818

Downloaded from Cranfield Library Services E-Repository 